\title{
NON-UNIFORM DEPENDENCE FOR HIGHER DIMENSIONAL CAMASSA-HOLM EQUATIONS IN BESOV SPACES
}

\author{
JINLU LI, WEI DENG, AND MIN LI
}

\begin{abstract}
In this paper, we investigate the dependence on initial data of solutions to higher dimensional Camassa-Holm equations. We show that the data-tosolution map is not uniformly continuous dependence in Besov spaces $B_{p, r}^{s}\left(\mathbb{R}^{d}\right), s>$ $\max \left\{1+\frac{d}{2}, \frac{3}{2}\right\}$.
\end{abstract}

\section{INTRODUCTION AND MAIN RESULT}

In the paper, we consider the following Cauchy problem of higher dimensional Camassa-Holm equations:

$$
\left\{\begin{array}{l}
\partial_{t} m+u \cdot \nabla m+\nabla u^{T} m+(\operatorname{div} u) m=0, \quad(t, x) \in \mathbb{R}^{+} \times \mathbb{R}^{d}, \\
m=(1-\Delta) u, \quad(t, x) \in \mathbb{R}^{+} \times \mathbb{R}^{d}, \\
u(0, x)=u_{0}, \quad x \in \mathbb{R}^{d},
\end{array}\right.
$$

where $u=\left(u_{1}, u_{2}, \cdots, u_{d}\right)$ denotes the velocity of the fluid, $m=\left(m_{1}, m_{2}, \cdots, m_{d}\right)$ represents the momentum, or we can write (1.1) in components,

$$
\partial_{t} m_{i}+\sum_{j=1}^{d} u_{j} \partial_{x_{j}} m_{i}+\sum_{j=1}^{d}\left(\partial_{x_{i}} u_{j}\right) m_{j}+m_{i} \sum_{j=1}^{d} \partial_{x_{j}} u_{j}=0, i=1,2, \cdots, d .
$$

While (1.1) is also called the Euler-Poincaré equations in the higher dimensional case $d \geq 1$. In particular, when $d=1$, the system (1.1) is the classical Camassa-Holm $(\mathrm{CH})$ equation, like the $\mathrm{KdV}$ equation, the $\mathrm{CH}$ equation describes the unidirectional propagation of waves at the free surface of shallow water under the influence of gravity $[4,5,16]$. It is completely integrable $[4,8]$, has a bi-Hamiltonian structure $[7$, 21 , and admits exact peaked solitons of the form $c e^{-|x-c t|}, c>0$, which are orbitally stable [18]. It is worth mentioning that the peaked solitons present the characteristic for the travelling water waves of greatest height and largest amplitude and arise as solutions to the free-boundary problem for incompressible Euler equations over a flat bed, cf. [10, 14, 15, 38]. The local well-posedness and ill-posedness for the Cauchy problem of the $\mathrm{CH}$ equation in Sobolev spaces and Besov spaces was discussed in $[11,12,19,20,22,32,37]$. The non-uniform dependence on initial data for the $\mathrm{CH}$ equation was studied in $[23,24,35]$. It was shown that there exist global strong solutions to the $\mathrm{CH}$ equation $[9,11,12]$ and finite time blow-up strong solutions to the $\mathrm{CH}$ equation $[9,11,12,13]$. The existence and uniqueness of global weak solutions to the $\mathrm{CH}$ equation were proved in $[17,39]$. The global conservative and dissipative solutions of $\mathrm{CH}$ equation were discussed in $[2,3]$.

2010 Mathematics Subject Classification. 35Q35.

Key words and phrases. Higher dimensional Camassa-Holm equations, Non-uniform continuous dependence, Besov spaces. 
For higher dimensional Camassa-Holm equations (hd-CH), or the so-called EulerPoincaré system (1.1) were first studied by Holm, Marsden, and Ratiu in 1998 as a framework for modeling and analyzing fluid dynamics [28, 29], particularly for nonlinear shallow water waves, geophysical fluids and turbulence modeling. Later, hd-CH equations have many further interpretations beyond fluid applications. For instance, in 2-D, it is exactly the same as the averaged template matching equation for computer vision [27]. Also, hd-CH equations have important applications in computational anatomy, it can be regarded as an evolutionary equation for a geodesic motion on a diffeomorphism group and it is associated with Euler-Poincaré reduction via symmetry (see, e.g, $[30,40]$ ).

The rigorous analysis of higher dimensional Camassa-Holm equations (1.1) with $d \geq 1$ was initiated by Chae and Liu [6] who obtained the local well-posedness in Hilbert spaces $m_{0} \in H^{s+\frac{d}{2}}, s \geq 2$ and also gave a blow-up criterion, zero $\alpha$ limit and the Liouville type theorem. Li, Yu and Zhai [31] proved that the solution to (1.1) with a large class of smooth initial data blows up in finite time or exists globally in time, which reveals the nonlinear depletion mechanism hidden in the Euler-Poincaré system. By means of the Littlewood-Paley theory, Yan and Yin [41] established the local existence and uniqueness in Besov spaces $B_{p, r}^{s}, s>\max \left\{\frac{3}{2}, 1+\frac{d}{p}\right\}$ and $s=1+\frac{d}{p}, 1 \leq p \leq 2 d, r=1$. Lately, Li and Yin [33] proved that the corresponding solution is continuous dependence for the initial data in Besov spaces. Inspired by $[25,26]$, Li, Dai and Zhu [34] show that the corresponding solution is not uniformly continuous dependence for the initial data in Sobolev spaces $H^{s}\left(\mathbb{R}^{d}\right), s>1+\frac{d}{2}$. For more results of higher dimensional Camassa-Holm equations, we refer the reads to see $[36,42]$.

In this paper, motivated by [35, 24], we will show that the solution map of (1.1) is not uniformly continuous depence in Besov space $B_{p, r}^{s}\left(\mathbb{R}^{d}\right), s>\max \left\{1+\frac{d}{2}, \frac{3}{2}\right\}$. Himonas-Misiolek [24] obtained the first result on the non-uniform dependence for the $\mathrm{CH}$ equation in $H^{s}(\mathbb{T})$ with $s \geq 2$ using explicitly constructed travelling wave solutions, these types of construction solutions are only suitable in Sobolev spaces. Instead, in this paper we constructed a more general initial data to obtain the nonuniform dependence in Besov spaces.

According to [41], we can transform (1.1) into the following form:

$$
\partial_{t} u+u \cdot \nabla u=Q(u, u)+R(u, u),
$$

where

$$
\begin{aligned}
Q(u, v)= & -(1-\Delta)^{-1} \operatorname{div}\left(\nabla u \nabla v+\nabla u(\nabla v)^{T}-(\nabla u)^{T} \nabla v\right. \\
& \left.-(\operatorname{div} u) \nabla v+\frac{1}{2} \mathbf{I}(\nabla u: \nabla v)\right), \\
R(u, v)= & -(1-\Delta)^{-1}((\operatorname{div} u) v+u \cdot \nabla v) .
\end{aligned}
$$

Then, we have the following result.

Theorem 1.1. Let $d \geq 2,1 \leq p, r \leq \infty$ and $s>\max \left\{1+\frac{d}{p}, \frac{3}{2}\right\}$. The data-tosolution map for higher dimensional Camassa-Holm equations (1.1) is not uniformly continuous from any bounded subset in $B_{p, r}^{s}$ into $\mathcal{C}\left([0, T] ; B_{p, r}^{s}\left(\mathbb{R}^{d}\right)\right)$. That is, there 
exists two sequences of solutions $u^{n}$ and $v^{n}$ such that

$$
\begin{aligned}
& \left\|u_{0}^{n}\right\|_{B_{p, r}^{s}\left(\mathbb{R}^{d}\right)}+\left\|v_{0}^{n}\right\|_{B_{p, r}^{s}\left(\mathbb{R}^{d}\right)} \lesssim 1, \quad \lim _{n \rightarrow \infty}\left\|u_{0}^{n}-v_{0}^{n}\right\|_{B_{p, r}^{s}\left(\mathbb{R}^{d}\right)}=0, \\
& \liminf _{n \rightarrow \infty}\left\|u^{n}(t)-v^{n}(t)\right\|_{B_{p, r}^{s}\left(\mathbb{R}^{d}\right)} \gtrsim t, \quad t \in\left[0, T_{0}\right],
\end{aligned}
$$

with small time $T_{0}$ for $T_{0} \leq T$.

Our paper is organized as follows. In Section 2, we give some preliminaries which will be used in the sequel. In Section 3, we give the proof of our main theorem.

Notations. Given a Banach space $X$, we denote its norm by $\|\cdot\|_{X}$. The symbol $A \lesssim B$ means that there is a uniform positive constant $c$ independent of $A$ and $B$ such that $A \leq c B$. Here

$$
\begin{aligned}
& \left(\nabla u^{T}\right)_{i, j}=\partial_{x_{i}} u^{j}, \quad(u \cdot \nabla v)_{i}=\sum_{k=1}^{d} u_{k} \partial_{x_{k}} u_{i}, \quad(\nabla u \nabla v)_{i j}=\sum_{k=1}^{d} \partial_{x_{i}} u_{k} \partial_{x_{k}} v_{j}, \\
& \nabla u: \nabla v=\sum_{i, j=1}^{d} \partial_{x_{i}} u_{j} \partial_{x_{i}} v_{j} .
\end{aligned}
$$

\section{LitTlewOOD-PALEY ANALYSiS}

In this section, we will recall some facts about the Littlewood-Paley decomposition, the nonhomogeneous Besov spaces and their some useful properties. For more details, the readers can refer to [1].

There exists a couple of smooth functions $(\chi, \varphi)$ valued in $[0,1]$, such that $\chi$ is supported in the ball $\mathcal{B} \triangleq\left\{\xi \in \mathbb{R}^{d}:|\xi| \leq \frac{4}{3}\right\}$, and $\varphi$ is supported in the ring $\mathcal{C} \triangleq\left\{\xi \in \mathbb{R}^{d}: \frac{3}{4} \leq|\xi| \leq \frac{8}{3}\right\}$. Moreover,

$$
\begin{gathered}
\forall \xi \in \mathbb{R}^{d}, \chi(\xi)+\sum_{j \geq 0} \varphi\left(2^{-j} \xi\right)=1, \\
\forall 0 \neq \xi \in \mathbb{R}^{d}, \sum_{j \in \mathbb{Z}} \varphi\left(2^{-j} \xi\right)=1, \\
\left|j-j^{\prime}\right| \geq 2 \Rightarrow \operatorname{Supp} \varphi\left(2^{-j} \cdot\right) \cap \operatorname{Supp} \varphi\left(2^{-j^{\prime}} \cdot\right)=\emptyset, \\
j \geq 1 \Rightarrow \operatorname{Supp} \chi(\cdot) \cap \operatorname{Supp} \varphi\left(2^{-j} \cdot\right)=\emptyset .
\end{gathered}
$$

Then, we can define the nonhomogeneous dyadic blocks $\Delta_{j}$ and nonhomogeneous low frequency cut-off operator $S_{j}$ as follows:

$$
\begin{gathered}
\Delta_{j} u=0, \text { if } j \leq-2, \quad \Delta_{-1} u=\chi(D) u=\mathcal{F}^{-1}(\chi \mathcal{F} u), \\
\Delta_{j} u=\varphi\left(2^{-j} D\right) u=\mathcal{F}^{-1}\left(\varphi\left(2^{-j}\right) \mathcal{F} u\right), \text { if } j \geq 0, \\
S_{j} u=\sum_{j^{\prime}=-\infty}^{j-1} \Delta_{j^{\prime}} u .
\end{gathered}
$$

Definition 2.1 ([1]). Let $s \in \mathbb{R}$ and $1 \leq p, r \leq \infty$. The nonhomogeneous Besov space $B_{p, r}^{s}$ consists of all tempered distribution $u$ such that

$$
\|u\|_{B_{p, r}^{s}\left(\mathbb{R}^{d}\right)} \triangleq\left\|\left(2^{j s}\left\|\Delta_{j} u\right\|_{L^{p}\left(\mathbb{R}^{d}\right)}\right)_{j \in \mathbb{Z}}\right\|_{\ell^{r}(\mathbb{Z})}<\infty .
$$

Then, we have the following product laws. 
Lemma $2.2([1])$. (1) For any $s>0$ and $1 \leq p, r \leq \infty$, there exists a positive constant $C=C(d, s, p, r)$ such that

$$
\|u v\|_{B_{p, r}^{s}\left(\mathbb{R}^{d}\right)} \leq C\left(\|u\|_{L^{\infty}\left(\mathbb{R}^{d}\right)}\|v\|_{B_{p, r}^{s}\left(\mathbb{R}^{d}\right)}+\|v\|_{L^{\infty}\left(\mathbb{R}^{d}\right)}\|u\|_{B_{p, r}^{s}\left(\mathbb{R}^{d}\right)}\right) .
$$

(2) Let $1 \leq p, r \leq \infty$ and $s>\max \left\{\frac{3}{2}, 1+\frac{d}{p}\right\}$. Then, we have

$$
\|u v\|_{B_{p, r}^{s-2}\left(\mathbb{R}^{d}\right)} \leq C\|u\|_{B_{p, r}^{s-1}\left(\mathbb{R}^{d}\right)}\|v\|_{B_{p, r}^{s-2}\left(\mathbb{R}^{d}\right)} .
$$

Lemma 2.3 (Theorem 3.38, [1] and Lemma 2.9, [33]). Let $1 \leq p, r \leq \infty$. Assume that

$$
\sigma>-d \min \left(\frac{1}{p}, \frac{1}{p^{\prime}}\right) \quad \text { or } \quad \sigma>-1-d \min \left(\frac{1}{p}, \frac{1}{p^{\prime}}\right) \quad \text { if } \quad \operatorname{div} v=0 .
$$

Assume that $f_{0} \in B_{p, r}^{\sigma}, g \in L^{1}\left(0, T ; B_{p, r}^{\sigma}\right)$, and $\nabla v$ belongs to $L^{1}\left(0, T ; B_{p, r}^{\sigma-1}\right)$ if $\sigma>1+\frac{d}{p}$ (or $\sigma=1+\frac{d}{p}$ and $r=1$ ) or to $L^{1}\left(0, T ; B_{p, r}^{\frac{d}{p}} \bigcap L^{\infty}\right)$ otherwise. If $f \in L^{\infty}\left(0, T ; B_{p, r}^{\sigma}\right) \cap \mathcal{C}\left([0, T] ; \mathcal{S}^{\prime}\right)$ solves the following linear transport equation:

$$
(T)\left\{\begin{array}{l}
\partial_{t} f+v \cdot \nabla f=g, \\
\left.f\right|_{t=0}=f_{0}
\end{array}\right.
$$

then there exists a constant $C=C(d, p, r, \sigma)$ such that the following statements hold:

$$
\sup _{s \in[0, t]}\|f(s)\|_{B_{p, r}^{\sigma}} \leq C e^{C V_{p}(v, t)}\left(\left\|f_{0}\right\|_{B_{p, r}^{\sigma}}+\int_{0}^{t}\|g(\tau)\|_{B_{p, r}^{s}} \mathrm{~d} \tau\right)
$$

with

$$
V_{p}(v, t)= \begin{cases}\int_{0}^{t}\|\nabla v(s)\|_{B_{p, \infty}^{\frac{d}{p}} \cap L^{\infty}} \mathrm{d} s, & \text { if } \sigma<1+\frac{d}{p}, \\ \int_{0}^{t}\|\nabla v(s)\|_{B_{p, r}^{\sigma}} \mathrm{d} s, & \text { if } \sigma=1+\frac{d}{p} \text { and } r>1, \\ \int_{0}^{t}\|\nabla v(s)\|_{B_{p, r}^{\sigma-1}} \mathrm{~d} s, & \text { if } \sigma>1+\frac{d}{p} \text { or }\left\{\sigma=1+\frac{d}{p} \text { and } r=1\right\} .\end{cases}
$$

If $f=v$, then for all $\sigma>0(\sigma>-1$, if $\operatorname{div} v=0)$, the estimate (2.2) holds with

$$
V_{p}(t)=\int_{0}^{t}\|\nabla v(s)\|_{L^{\infty}} \mathrm{d} s .
$$

\section{NON-UNIFORM CONTINUOUS DEPENDENCE}

In this section, motivated by [35], we will give the proof of our main theorem. First, we can construct a sequence initial data $u_{0}^{n}=f_{n}$, which can approximate to the solution $\mathbf{S}_{t}\left(u_{0}^{n}\right)$. Lately, we can construct a sequence initial data $v_{0}^{n}=f_{n}+g_{n}$ and can approximate to the solution $\mathbf{S}_{t}\left(v_{0}^{n}\right)$ by $v_{0}^{n}+t g_{n} \cdot \nabla f_{n}$. Finally, by the precious steps, we can conclude that the solution map is not uniformly continuous. In order to state our main result, we first recall the following local-in-time existence of strong solutions to (1.1) in [41]:

Lemma $3.1([41])$. For $1 \leq p, r \leq \infty$ and $s>\max \left\{1+\frac{d}{p}, \frac{3}{2}\right\}$ and initial data $u_{0} \in$ $B_{p, r}^{s}\left(\mathbb{R}^{d}\right)$, there exists a time $T=T\left(s, p, r, d,\left\|u_{0}\right\|_{B_{p, r}^{s}\left(\mathbb{R}^{d}\right)}\right)>0$ such that the system (1.1) have a unique solution $u \in \mathcal{C}\left([0, T] ; B_{p, r}^{s}\left(\mathbb{R}^{d}\right)\right)$. Moreover, for all $t \in[0, T]$, there holds

$$
\|u(t)\|_{B_{p, r}^{s}\left(\mathbb{R}^{d}\right)} \leq C\left\|u_{0}\right\|_{B_{p, r}^{s}\left(\mathbb{R}^{d}\right)} .
$$


Corollary 3.2. Let $1 \leq p, r \leq \infty$ and $s>\max \left\{1+\frac{d}{p}, \frac{3}{2}\right\}$. Assume that $u \in$ $\mathcal{C}\left([0, T] ; B_{p, r}^{s}\right)$ be the solution of the system (1.1). Then, we have for all $t \in[0, T]$,

$$
\|u(t)\|_{B_{p, r}^{s-1}\left(\mathbb{R}^{d}\right)} \leq\left\|u_{0}\right\|_{B_{p, r}^{s-1}\left(\mathbb{R}^{d}\right)} e^{C \int_{0}^{t}\|u(\tau)\|_{B_{p, r}^{s}\left(\mathbb{R}^{d}\right)} \mathrm{d} \tau}
$$

and

$$
\|u(t)\|_{B_{p, r}^{s+1}\left(\mathbb{R}^{d}\right)} \leq\left\|u_{0}\right\|_{B_{p, r}^{s+1}\left(\mathbb{R}^{d}\right)} e^{C \int_{0}^{t}\|u(\tau)\|_{B_{p, r}^{s}\left(\mathbb{R}^{d}\right)} \mathrm{d} \tau} .
$$

Proof. The results can easily deduce from Lemma 2.3 and Gronwall's inequality. Here, we omit it.

Now, we give the details of the proof to our theorem.

Proof of the main theorem. Letting $\hat{\phi}$ be a $C_{0}(\mathbb{R})$ such that

$$
\hat{\phi}(x)= \begin{cases}1, & |x| \leq \frac{1}{4^{d}}, \\ 0, & |x| \geq \frac{1}{2^{d}} .\end{cases}
$$

First, we choose the velocity $u_{0}^{n}$ having the following form:

$$
u_{0}^{n}=\left(f_{n}, 0, \cdots, 0\right) \text {, }
$$

with

$$
f_{n}(x)=2^{-n s} \phi\left(x_{1}\right) \sin \left(\frac{17}{12} x_{1}\right) \phi\left(x_{2}\right) \cdots \phi\left(x_{d}\right), \quad n \in \mathbb{Z} .
$$

An easy computation gives that

$$
\hat{f}_{n}=2^{-n s-1} i\left[\hat{\phi}\left(\xi_{1}+\frac{17}{12} 2^{n}\right)-\hat{\phi}\left(\xi_{1}-\frac{17}{12} 2^{n}\right)\right] \hat{\phi}\left(\xi_{2}\right) \cdots \hat{\phi}\left(\xi_{d}\right),
$$

which implies

$$
\operatorname{supp} \hat{f}_{n} \subset\left\{\xi \in \mathbb{R}^{d}: \frac{17}{12} 2^{n}-\frac{1}{2} \leq|\xi| \leq \frac{17}{12} 2^{n}+\frac{1}{2}\right\} .
$$

Then, we deduce that

$$
\Delta_{j}\left(f_{n}\right)= \begin{cases}f_{n}, & j=n, \\ 0, & j \neq n .\end{cases}
$$

On account of Definition 2.1, we can show that for $k \in \mathbb{R}$,

$$
\left\|u_{0}^{n}\right\|_{B_{p, r}^{s+k}} \leq C 2^{k n} \text {. }
$$

Let $u^{n}$ be the solution of (1.2) with initial data $u_{0}^{n}$. Then, we have the following estimate between $u_{0}^{n}$ and $u^{n}$.

Lemma 3.3. Let $\varepsilon_{s}=\frac{1}{2} \min \left\{s-1-\frac{d}{p}, s-\frac{3}{2}, 1\right\}$, then there holds

$$
\left\|u^{n}-u_{0}^{n}\right\|_{L_{T}^{\infty}\left(B_{p, r}^{s}\right)} \leq C 2^{-n \varepsilon_{s}}
$$

where $T \simeq 1$.

Proof. By the well-posedness result (see Lemma 3.1), the solution $u^{n}$ belong to $\mathcal{C}\left([0, T] ; B_{p, r}^{s}\right)$ and have lifespan $T \simeq 1$. In fact, it is easy to show that for $k>-s$,

$$
\left\|u^{n}\right\|_{L_{T}^{\infty}\left(B_{p, r}^{s+k}\right)} \leq C 2^{k n} \text {. }
$$


Since $s-\varepsilon_{s}-1>\frac{d}{p}$, then we have $\|f\|_{L^{\infty}} \leq C\|f\|_{B_{p, r}^{s-1-\varepsilon_{s}}}$. Hence, we obtain

$$
\begin{aligned}
\left\|u^{n}-u_{0}^{n}\right\|_{B_{p, r}^{s}} & \leq \int_{0}^{t}\left\|\partial_{\tau} u^{n}\right\|_{B_{p, r}^{s}} \mathrm{~d} \tau \\
& \leq\left\|u^{n}\right\|_{L^{\infty}}\left\|u^{n}\right\|_{B_{p, r}^{s+1}}+\left\|\nabla u^{n}\right\|_{L^{\infty}}\left\|u^{n}\right\|_{B_{p, r}^{s}} \\
& \leq\left\|u^{n}\right\|_{B_{p, r}^{s-1-\varepsilon_{s}}}\left\|u^{n}\right\|_{B_{p, r}^{s+1}}+\left\|\nabla u^{n}\right\|_{B_{p, r}^{s-\varepsilon_{s}}}\left\|u^{n}\right\|_{B_{p, r}^{s}} \\
& \leq 2^{-n \varepsilon_{s} .}
\end{aligned}
$$

Now, we choose the velocity $v_{0}^{n}$ having the following form:

$$
v_{0}^{n}=\left(f_{n}+g_{n}, 0, \cdots, 0\right),
$$

with

$$
g_{n}(x)=2^{-n} \phi\left(x_{1}\right) \phi\left(x_{2}\right) \cdots \phi\left(x_{d}\right), \quad n \in \mathbb{Z} .
$$

Direct calculation shows that for $k \geq-1$,

$$
\left\|v_{0}^{n}\right\|_{B_{p, r}^{s+k}} \leq C 2^{k n}, \quad\left\|v_{0}^{n}, \nabla v_{0}^{n}\right\|_{L^{\infty}} \leq C\left(2^{-n}+2^{-(s-1) n}\right) .
$$

Let $v^{n}$ be the solution of (1.2) with initial data $v_{0}^{n}$. Then, we have the following estimate between $v_{0}^{n}$ and $v^{n}$.

Lemma 3.4. Let $\varepsilon_{s}=\frac{1}{2} \min \left\{s-1-\frac{d}{p}, s-\frac{3}{2}, 1\right\}$, then there holds for $t \leq T$

$$
\left\|v^{n}(t, \cdot)-v_{0}^{n}+t v_{0}^{n} \cdot \nabla v_{0}^{n}\right\|_{B_{p, r}^{s}} \leq C 2^{-n \varepsilon_{s}}+C t^{2} .
$$

Proof. By the well-posedness result (see Lemma 3.1), the solution $v^{n}$ belong to $\mathcal{C}\left([0, T] ; B_{p, r}^{s}\right)$ and have common lifespan $T \simeq 1$. It is easy to check that

$$
\left\|v_{0}^{n}, V_{0}^{n}, v^{n}\right\|_{B_{p, r}^{s+k}} \leq C 2^{k n}, \quad \text { for } \quad k=-1,0,1 .
$$

For simplicity, we denote $w_{n}=v^{n}-v_{0}^{n}-t V_{0}^{n}$ with $V_{0}^{n}=-v_{0}^{n} \cdot \nabla v_{0}^{n}$, then we can deduce that

$$
\begin{aligned}
\partial_{t} w_{n}+v^{n} \cdot \nabla w_{n}= & -t\left(V_{0}^{n} \cdot \nabla v_{0}^{n}+v_{0}^{n} \cdot \nabla V_{0}^{n}\right)-t^{2} V_{0}^{n} \cdot \nabla V_{0}^{n} \\
& -w_{n} \cdot \nabla\left(v_{0}^{n}+t V_{0}^{n}\right)+Q\left(v^{n}, v^{n}\right)+R\left(v^{n}, v^{n}\right) .
\end{aligned}
$$

From Lemma 2.2, we have

$$
\left\|R\left(v^{n}, v^{n}\right)\right\|_{B_{p, r}^{s-1}} \leq C\left\|v_{n}\right\|_{B_{p, r}^{s-1}}^{2} \leq C 2^{-2 n}
$$

and

$$
\left\|R\left(v^{n}, v^{n}\right)\right\|_{B_{p, r}^{s}} \leq C\left\|\nabla v_{n}\right\|_{B_{p, r}^{s-1}}\left\|v_{n}\right\|_{B_{p, r}^{s-1}} \leq C 2^{-n} .
$$

For the term $Q\left(v^{n}, v^{n}\right)$, we need decompose it as follows:

$$
Q\left(v^{n}, v^{n}\right)=Q\left(w^{n}, v^{n}\right)+Q\left(v_{0}^{n}, w^{n}\right)+Q\left(v_{0}^{n}, t V_{0}^{n}\right)+Q\left(t V_{0}^{n}, v^{n}\right)+Q\left(v_{0}^{n}, v_{0}^{n}\right) .
$$


Then, combining (3.3) and (3.6), we see that

$$
\begin{aligned}
\partial_{t} w_{n}+v^{n} \cdot \nabla w_{n}= & \underbrace{-t\left(V_{0}^{n} \cdot \nabla v_{0}^{n}+v_{0}^{n} \cdot \nabla V_{0}^{n}+t V_{0}^{n} \cdot \nabla V_{0}^{n}-Q\left(V_{0}^{n}, v^{n}\right)-Q\left(v_{0}^{n}, V_{0}^{n}\right)\right)}_{I} \\
& \underbrace{-w_{n} \cdot \nabla\left(v_{0}^{n}+t V_{0}^{n}\right)+Q\left(w^{n}, v^{n}\right)+Q\left(v_{0}^{n}, w^{n}\right)}_{I I} \\
& +\underbrace{Q\left(v_{0}^{n}, v_{0}^{n}\right)+R\left(v^{n}, v^{n}\right)}_{I I I} .
\end{aligned}
$$

By Lemma 2.2 and (3.2), we obtain

$$
\|I(t)\|_{B_{p, r}^{s-1}} \leq C\left\|v^{n}, v_{0}^{n}, V_{0}^{n}\right\|_{B_{p, r}^{s-1}}\left\|v^{n}, v_{0}^{n}, V_{0}^{n}\right\|_{B_{p, r}^{s}} \leq C t 2^{-n}
$$

and

$$
\|I I(t)\|_{B_{p, r}^{s-1}} \leq C\left\|w_{n}\right\|_{B_{p, r}^{s-1}}\left\|v^{n}, v_{0}^{n}, V_{0}^{n}\right\|_{B_{p, r}^{s}} \leq C\left\|w_{n}\right\|_{B_{p, r}^{s-1}} .
$$

For the term $I I I(t)$, we just estimate $Q\left(v_{0}^{n}, v_{0}^{n}\right)$. It follows from Lemma 2.2 and (3.1) that

$$
\begin{aligned}
\left\|Q\left(v_{0}^{n}, v_{0}^{n}\right)\right\|_{B_{p, r}^{s-1}} & \leq C\left\|Q\left(v_{0}^{n}, v_{0}^{n}\right)\right\|_{B_{p, r}^{s-\frac{1}{2}}} \leq C\left\|v_{0}^{n}\right\|_{B_{p, r}^{s-\frac{1}{2}}}\left\|v_{0}^{n}, \nabla v_{0}^{n}\right\|_{L^{\infty}} \\
& \leq C 2^{-\frac{1}{2} n}\left(2^{-n(s-1)}+2^{-n}\right) \leq C 2^{-n\left(1+\varepsilon_{s}\right)},
\end{aligned}
$$

which along with (3.4) implies

$$
\|I I I(t)\|_{B_{p, r}^{s-1}} \leq C 2^{-n\left(1+\varepsilon_{s}\right)} .
$$

Thus, we infer from Lemma 2.3 and (3.7)-(3.9) that

$$
\left\|w_{n}\right\|_{B_{p, r}^{s-1}} \leq \int_{0}^{t}\left\|w_{n}\right\|_{B_{p, r}^{s-1}} \mathrm{~d} \tau+C t^{2}+C 2^{-n\left(1+\varepsilon_{s}\right)}
$$

which yields

$$
\left\|w_{n}\right\|_{B_{p, r}^{s-1}} \leq C 2^{-n} t^{2}+C 2^{-n\left(1+\varepsilon_{s}\right)} .
$$

Now, we can tackle with the $B_{p, r}^{s}$ norm of $w_{n}$. According to Lemma 2.2 and (3.2), we have

$$
\|I(t)\|_{B_{p, r}^{s}} \leq C\left\|v^{n}, v_{0}^{n}, V_{0}^{n}\right\|_{B_{p, r}^{s-1}}\left\|v^{n}, v_{0}^{n}, V_{0}^{n}\right\|_{B_{p, r}^{s+1}}+C\left\|v^{n}, v_{0}^{n}, V_{0}^{n}\right\|_{B_{p, r}^{s}}^{2} \leq C t,
$$

and

$$
\begin{aligned}
\|I I(t)\|_{B_{p, r}^{s}} & \leq C\left\|w_{n}\right\|_{B_{p, r}^{s}}\left\|v^{n}, v_{0}^{n}, V_{0}^{n}\right\|_{B_{p, r}^{s}}+C\left\|w_{n}\right\|_{B_{p, r}^{s+1}}\left\|v^{n}, v_{0}^{n}, V_{0}^{n}\right\|_{B_{p, r}^{s-1}} \\
& \leq C\left\|w_{n}\right\|_{B_{p, r}^{s}}+C 2^{n}\left\|w_{n}\right\|_{B_{p, r}^{s-1}}
\end{aligned}
$$

From Lemma 2.2 and (3.1), we obtain

$$
\begin{aligned}
\left\|Q\left(v_{0}^{n}, v_{0}^{n}\right)\right\|_{B_{p, r}^{s}} & \leq C\left\|v_{0}^{n}\right\|_{B_{p, r}^{s}}\left\|v_{0}^{n}, \nabla v_{0}^{n}\right\|_{L^{\infty}} \\
& \leq C\left\|v_{0}^{n}\right\|_{B_{p, r}^{s}}\left\|v_{0}^{n}, \nabla v_{0}^{n}\right\|_{B_{p, r}^{s-\varepsilon_{s}}} \leq C 2^{-n \varepsilon_{s}},
\end{aligned}
$$

which along with (3.5) leads to

$$
\|I I I(t)\|_{B_{p, r}^{s}} \leq C 2^{-n \varepsilon_{s}} .
$$


According to Lemma 2.3 and (3.10), (3.11)-(3.13), we have

$$
\begin{aligned}
\left\|w_{n}\right\|_{B_{p, r}^{s}} & \leq C \int_{0}^{t}\left\|w_{n}\right\|_{B_{p, r}^{s}} \mathrm{~d} \tau+\int_{0}^{t}\left\|w_{n}\right\|_{B_{p, r}^{s-1}}\left\|v_{0}^{n}, V_{0}^{n}\right\|_{B_{p, r}^{s+1}} \mathrm{~d} \tau+C t^{2}+C 2^{-n \varepsilon_{s}} \\
& \leq C \int_{0}^{t}\left\|w_{n}\right\|_{B_{p, r}^{s}} \mathrm{~d} \tau+C t^{2}+C 2^{-n \varepsilon_{s}},
\end{aligned}
$$

which gives

$$
\left\|w_{n}\right\|_{B_{p, r}^{s}} \leq C t^{2}+C 2^{-n \varepsilon_{s}} .
$$

Finally, we prove the result of Theorem 1.1. It is easy to show that

$$
\left\|u_{0}^{n}-v_{0}^{n}\right\|_{B_{p, r}^{s}} \leq\left\|g_{n}\right\|_{B_{p, r}^{s}} \leq C 2^{-n}
$$

which tend to 0 for $n$ tends to infinity. For the solution, using of triangle inequality,

$$
\begin{aligned}
& \left\|u^{n}-v^{n}\right\|_{B_{p, r}^{s}} \\
= & \left\|\left(u^{n}-u_{0}^{n}\right)-\left(v^{n}-v_{0}^{n}+t v_{0}^{n} \cdot \nabla v_{0}^{n}\right)+u_{0}^{n}-v_{0}^{n}+t v_{0}^{n} \cdot \nabla v_{0}^{n}\right\|_{B_{p, r}^{s}} \\
\geq & \left\|t v_{0}^{n} \cdot \nabla v_{0}^{n}\right\|_{B_{p, r}^{s}}-\left\|u_{0}^{n}-v_{0}^{n}\right\|_{B_{p, r}^{s}}-\left\|u^{n}-u_{0}^{n}\right\|_{B_{p, r}^{s}}-\left\|v^{n}-v_{0}^{n}+t v_{0}^{n} \cdot \nabla v_{0}^{n}\right\|_{B_{p, r}^{s}} .
\end{aligned}
$$

Thanks for Lemma 3.3 and Lemma 3.4, we have

$$
\left\|u^{n}-v^{n}\right\|_{B_{p, r}^{s}} \geq\left\|t v_{0}^{n} \cdot \nabla v_{0}^{n}\right\|_{B_{p, r}^{s}}-C t^{2}-C 2^{-n \varepsilon_{s}} .
$$

As $\left(v_{0}^{n} \cdot \nabla v_{0}^{n}\right)_{i}=0$ for $i=2, \cdots d$, and

$$
\left(v_{0}^{n} \cdot \nabla v_{0}^{n}\right)_{1}=f_{n} \cdot \nabla f_{n}+f_{n} \cdot \nabla g_{n}+g_{n} \cdot \nabla f_{n}+g_{n} \cdot \nabla g_{n} .
$$

By the definition of $f_{n}$ and $g_{n}$, we have

$$
\begin{aligned}
& \left\|f_{n} \cdot \nabla f_{n}, g_{n} \cdot \nabla g_{n}, f_{n} \cdot \nabla g_{n}\right\|_{B_{p, r}^{s}} \\
\leq & C\left\|f_{n}, g_{n}\right\|_{L^{\infty}}\left\|f_{n}, g_{n}\right\|_{B_{p, r}^{s}}+C\left\|\nabla f_{n}, g_{n}\right\|_{L^{\infty}}\left\|f_{n}, g_{n}\right\|_{B_{p, r}^{s}} \\
\leq & C 2^{-n \varepsilon_{s}}
\end{aligned}
$$

which along with (3.15) and (3.16) implies

$$
\left\|u^{n}-v^{n}\right\|_{B_{p, r}^{s}} \geq c t\left\|g_{n} \cdot \nabla f_{n}\right\|_{B_{p, r}^{s}}-C t^{2}-C 2^{-n \varepsilon_{s}} .
$$

Noticing that

$$
\begin{aligned}
\left\|g_{n} \cdot \nabla f_{n}\right\|_{B_{p, r}^{s}} & =2^{n s}\left\|g_{n} \cdot \nabla f_{n}\right\|_{L^{p}} \\
& \geq\left\|\phi^{2}\left(x_{1}\right) \cos \left(\frac{17}{12} 2^{n} x_{1}\right)\right\|_{L^{p}}\|\phi\|_{L^{p}}^{2(d-1)}-C 2^{-n \varepsilon_{s}} .
\end{aligned}
$$

Substituting (3.18) into (3.17), we obtain

$$
\left\|u^{n}-v^{n}\right\|_{B_{p, r}^{s}} \geq c t-C t^{2}-C 2^{-n \varepsilon_{s}} .
$$

Restricting $T_{0}$ to a small range, by choosing another $c$ we have

$$
\left\|u^{n}(t)-v^{n}(t)\right\|_{B_{p, r}^{s}\left(\mathbb{R}^{d}\right)} \geq c t, \quad t \in\left[0, T_{0}\right] .
$$

Letting $n$ go to $\infty$, then (3.19) together with (3.14) complete the proof of Theorem 1.1.

Acknowledgements. J. Li is supported by the National Natural Science Foundation of China (Grant No.11801090). 


\section{REFERENCES}

[1] H. Bahouri, J. Y. Chemin and R. Danchin, Fourier Analysis and Nonlinear Partial Differential Equations, Grundlehren der Mathematischen Wissenschaften, vol. 343, Springer-Verlag, Berlin, Heidelberg, 2011.

[2] A. Bressan and A. Constantin, Global conservative solutions of the Camassa-Holm equation, Arch. Ration. Mech. Anal., 183 (2007), 215-239.

[3] A. Bressan and A. Constantin, Global dissipative solutions of the Camassa-Holm equation, Anal. Appl., 5 (2007), 1-27.

[4] R. Camassa and D. D. Holm, An integrable shallow water equation with peaked solitons, Phys. Rev. Lett., 71 (1993), 1661-1664.

[5] R. Camassa, D. Holm and J. Hyman, A new integrable shallow water equation, Adv. Appl. Mech., 31 (1994), 1-33.

[6] D. Chae and J. Liu, Blow-up, zero a limit and the Liouville type theorem for the Euler-Poincaré equations, Comm. Math. Phys., 314 (2012), 671-687.

[7] A. Constantin, The Hamiltonian structure of the Camassa-Holm equation, Exposition. Math., 15 (1997), 53-85.

[8] A. Constantin, On the scattering problem for the Camassa-Holm equation, R. Soc. Lond. Proc. Ser. A Math. Phys. Eng. Sci., 457 (2001), 953-970.

[9] A. Constantin, Existence of permanent and breaking waves for a shallow water equation: a geometric approach, Ann. Inst. Fourier (Grenoble), 50 (2000), 321-362.

[10] A. Constantin, The trajectories of particles in Stokes waves, Invent. Math., 166 (2006), 523535 .

[11] A. Constantin and J. Escher, Global existence and blow-up for a shallow water equation, Ann. Scuola Norm. Sup. Pisa Cl. Sci. (4), 26 (1998), 303-328.

[12] A. Constantin and J. Escher, Well-posedness, global existence, and blowup phenomena for a periodic quasi-linear hyperbolic equation, Comm. Pure Appl. Math., 51 (1998), 475-504.

[13] A. Constantin and J. Escher, Wave breaking for nonlinear nonlocal shallow water equations, Acta Math., 181 (1998), 229-243.

[14] A. Constantin and J. Escher, Particle trajectories in solitary water waves, Bull. Amer. Math. Soc., 44 (2007), 423-431.

[15] A. Constantin and J. Escher, Analyticity of periodic traveling free surface water waves with vorticity, Ann. of Math., 173 (2011), 559-568.

[16] A. Constantin and D. Lannes, The hydrodynamical relevance of the Camassa-Holm and Degasperis-Procesi equations, Arch. Ration. Mech. Anal., 192 (2009), 165-186.

[17] A. Constantin and L. Molinet Global weak solutions for a shallow water equation, Comm. Math. Phys., 211 (2000), 45-61.

[18] A. Constantin and W. A. Strauss, Stability of peakons, Comm. Pure Appl. Math., 53 (2000), 603-610.

[19] R. Danchin, A few remarks on the Camassa-Holm equation. Differential Integral Equations, 14 (2001), 953-988.

[20] R. Danchin, A note on well-posedness for Camassa-Holm equation, J. Differential Equations, 192 (2003), 429-444.

[21] A. Fokas and B. Fuchssteiner, Symplectic structures, their Bäcklund transformation and hereditary symmetries, Phys. D, 4 (1981/82), 47-66.

[22] Z. Guo, X. Liu, M. Luc and Z. Yin, Ill-posedness of the Camassa-Holm and related equations in the critical space, J. Differential Equations, 266 (2019), 1698-1707.

[23] A. Himonas and C. Kenig C 2009 Non-uniform dependence on initial data for the CH equation on the line, Diff. Integral Eqns, 22 (2009), 201-224.

[24] A. Himonas, C. Kenig and Misiolek Non-uniform dependence for the periodic CH equation, Commun. Partial Diff. Eqns, 35 (2010), 1145-1162.

[25] A. Himonas and C. Holliman, The Cauchy problem for the Novikov equation, Nonlinearity, 25 (2012), 449-479.

[26] A. Himonas and G. Misiołek, Non-uniform dependence on initial data of solutions to the Euler equations of hydrodynamics, Comm. Math. Phys., 296 (2010), 285-301. 
[27] A.N. Hirani, J.E. Marsden, J. Arvo, Averaged template matching equations, Lecture Notes in Computer Science, EMMCVPR, Berlin Heidelberg New York: Springer, 2134 (2001), 528-543.

[28] D.D. Holm, J.E. Marsden, T.S. Ratiu, Euler-Poincaré models of ideal fluids with nonlinear disper-sion, Phys. Rev. Lett., 80 (1998),4173-4177.

[29] D.D. Holm, J.E. Marsden, T.S. Ratiu, Euler-Poincaré equations and semi-direct products with applica-tions to continuum theories, Adv. in Math., 137(1998),1-81.

[30] D.D. Holm, T. Schmah and C. Stoica, Geometric Mechanics and Symmetry: From Finite to Infinite Dimensions, vol.12, Oxford University Press, 2009.

[31] D. Li, X. Yu and Z. Zhai, On the Euler-Poincare equation with non-zero dispersion, Arch. Ration. Mech. Anal., 210 (2013), 955-974.

[32] J. Li and Z. Yin, Remarks on the well-posedness of Camassa-Holm type equations in Besov spaces, J. Differential Equations, 261 (2016), 6125-6143.

[33] J. Li and Z. Yin, Well-posedness and analytic solutions of the two-component Euler-Poincaré system, Monatsh. Math., 183 (2017), 509-537.

[34] J. Li, L. Dai and W. Zhu, Non-uniform continuous dependence on initial data of solutions to the Euler-Poincaré system, J. Math. Phys., 60 (2019), 111510, 9 pp.

[35] J. Li, Y, Yu and W. Zhu, Non-uniform dependence on initial data for the Camassa-Holm equation in Besov spaces, arXiv:2001.00290.

[36] W. Luo and Z. Yin, Blow-up phenomena, ill-posedness and peakon solutions for the periodic Euler-Poincaré equations, J. Differential Equations, 268 (2020), 1307-1325.

[37] G. Rodríguez-Blanco, On the Cauchy problem for the Camassa-Holm equation, Nonlinear Anal., 46 (2001), 309-327.

[38] J. F. Toland, Stokes waves, Topol. Methods Nonlinear Anal., 7 (1996), 1-48.

[39] Z. Xin and P. Zhang, On the weak solutions to a shallow water equation, Comm. Pure Appl. Math., 53 (2000), 1411-1433.

[40] L. Younes, Shapes and Diffeomorphisms Vol. 171, Berlin: Springer, 2010.

[41] K. Yan and Z. Yin, On the initial value problem for higher dimensional Camassa-Holm equations, Discrete Contin. Dyn. Syst., 35 (2015), 1327-1358.

[42] Y. Zhao, M. Yang and Y. Li, Non-uniform dependence for the periodic higher dimensional Camassa-Holm equations, J. Math. Anal. Appl., 461 (2018), 59-73.

School of Mathematics and Computer Sciences, Gannan Normal University, Ganzhou 341000, CHINA

E-mail address: lijinlu@gnnu.edu.cn

School of Mathematics and Computer Sciences, Gannan Normal University, Ganzhou 341000, CHINA

E-mail address: dwhh0406@163.com

School of intormation Management, Jiangxi University Of Finance and EconomICS 330032, CHINA

E-mail address: limin@jxufe.edu.cn 